\title{
The environment of HIl galaxies revisited
}

\author{
E. Koulouridis ${ }^{1}$, M. Plionis ${ }^{2,3}$, R. Chávez ${ }^{3}$, E. Terlevich ${ }^{3}$, R. Terlevich ${ }^{3,4}$, F. Bresolin ${ }^{5}$, and S. Basilakos ${ }^{6}$ \\ 1 Institute for Astronomy \& Astrophysics, Space Applications \& Remote Sensing, National Observatory of Athens, \\ Palaia Penteli, 15236 Athens, Greece \\ e-mail: ekoulour@noa.gr \\ 2 Physics Department of Aristotle University of Thessaloniki, University Campus, 54124 Thessaloniki, Greece \\ 3 Instituto Nacional de Astrofísica Optica y Electrónica, CP 72840 Puebla, Mexico \\ 4 Institute of Astronomy, University of Cambridge, Madingley Rd, CB3 OHA, Cambridge \\ ${ }^{5}$ Institute for Astronomy of the University of Hawaii, 2680 Woodlawn Drive, 96822 Honolulu, HI, USA \\ 6 Academy of Athens Research Center for Astronomy \& Applied Mathematics, Soranou Efessiou 4, 11-527 Athens, Greece
}

Received 14 December 2012 / Accepted 26 March 2013

\begin{abstract}
We present a study of the close $\left(\lesssim 200 h_{75}^{-1} \mathrm{kpc}\right)$ environment of 110 relatively local $(z \lesssim 0.16)$ HII galaxies, selected from the Sloan Digital Sky Survey (SDSS; DR7). We use available spectroscopic and photometric redshifts to investigate the presence of a close and possibly interacting companion galaxy. Our aim is to compare the physical properties of isolated and interacting HII galaxies and investigate possible systematic effects in their use as cosmological probes. We find that interacting HII galaxies tend to be more compact, less luminous and have a lower velocity dispersion than isolated ones, in agreement with previous studies on smaller samples. However, as we verify, these environmental differences do not affect the cosmologically important $L_{\mathrm{H} \beta}-\sigma$ correlation of the HII galaxies.
\end{abstract}

Key words. galaxies: star formation - galaxies: starburst - galaxies: evolution - large-scale structure of Universe galaxies: interactions

\section{Introduction}

HII galaxies are compact dwarf objects with massive star formation bursts. They are characterized by a high luminosity per unit mass, concentrated mostly in a few strong emission lines in the optical rest frame, a fact that makes them visible at very large redshifts. This, together with the observed correlation between the luminosity of recombination lines, e.g. $L(\mathrm{H} \beta)$ and the ionized gas velocity dispersion $\sigma$ (see Terlevich \& Melnick 1981; Melnick et al. 1988; Fuentes-Masip et al. 2000; Telles et al. 2001; Bosch et al. 2002; Siegel et al. 2005; Bordalo \& Telles 2011) renders them alternative cosmological distance probes. In Plionis et al. (2011) we presented a thorough investigation of the viability of using HII galaxies to constrain the dark energy equation of state and they indeed appear to be a prominent cosmological probe (see also Melnick et al. 2000; Siegel et al. 2005). This was clearly verified by using them to estimate the Hubble constant, finding a value $H_{0}=74.3 \pm 4.3 \mathrm{~km} \mathrm{~s}^{-1} \mathrm{Mpc}^{-1}$ (Chávez et al. 2012), in excellent agreement with, and independently confirming, the most recent SNIa based results (Riess et al. 2011; Freedman et al. 2012).

The cosmological importance of the HII galaxies forces us to investigate all possible sources of systematic effects that could affect the observed $L(\mathrm{H} \beta)-\sigma$ correlation. One such systematic could well be related to the effects of the close environment of HII galaxies. It is widely accepted that interactions between two galaxies are capable of triggering starburst events by driving gas and molecular clouds from the outskirts toward the center of each galaxy (e.g. Li et al. 2008; Ellison et al. 2008; Ideue et al. 2012). These events enhance greatly the star formation rate of the galaxies and consequently they appear intensely blue because of their abundance in young stars. The idea of the interactions-starburst connection was greatly supported by the studies of ultraluminous infrared galaxies (e.g. Sanders \& Mirabel 1996; Surace et al. 1998) which were found to be strongly interacting and by definition highly star forming. In addition, supportive evidence of interaction-induced star formation, at lower infrared luminosities, was given by Koulouridis et al. (2006).

On the other hand, regarding HII galaxies, previous studies on the environmental dependence of their properties concluded that they appear to be less clustered than "normal" galaxies (e.g. Iovino et al. 1988; Loveday et al. 1999; Telles \& Maddox 2000) and to have a deficiency in bright neighbors (Campos-Aguilar \& Moles 1991; Campos-Aguilar et al. 1993). These results questioned the efficiency of interactions as the starburst's triggering mechanism at least for the specific objects. However, Noeske et al. (2006) argued that since $~ 30 \%$ of their sample's star forming dwarf galaxies (SFDGs) have mostly dwarf neighbors, this percentage is a lower limit because of the poor completness of the $\mathrm{NED}^{1}$, which they used to conduct their search. In addition, numerical simulations (Bekki 2008) also showed that some compact star forming galaxies can be the result of dwarfdwarf merging. We should note, however, that although faint dwarf neighbors (in projection) were also probably found in a sample of SFDGs by Brosch et al. (2006), they were considered as noninteracting, because of their large distance, and rather as a sign of synchronized star formation over a large area.

\footnotetext{
1 NASA/IPAC Extragalactic Database is operated by the Jet Propulsion Laboratory, California Institute of Technology, under contract with the National Aeronautics and Space Administration.
} 
Interestingly, Telles \& Terlevich (1995) found, by investigating the environment of 51 HII galaxies, that only $\sim 10 \%$ of their sample had a luminous neighboring galaxy and they tend to be those of lower $\mathrm{H} \beta$ luminosity, lower velocity dispersion and regular morphology, while on the contrary the majority of luminous objects seem to be irregular, disturbed and isolated (see also Telles et al. 1997). Similar results were also reported in Vilchez (1995) where the SFDGs in low density regions have larger $\mathrm{H} \beta$ equivalent widths and higher $\mathrm{H} \beta$ luminosities.

Most of these studies however, investigated the effects of what could be called the large scale environment, since their radial limit for the identification of a possible neighbor was at least $1 h_{75}^{-1} \mathrm{Mpc}$. In addition they were relatively "shallow" because of the low magnitude limit of the available redshift surveys at the time and as a result they were sensitive only to the more luminous and massive neighbors.

The aim of the present study is to investigate the environment of a larger sample of 128 HII galaxies, selected from the SDSS, which enables us to perform a consistent environmental analysis using a fixed magnitude difference between the HII galaxies and their neighbors, while reaching fainter magnitudes. More importantly, we would like to investigate the already mentioned trend reported by Telles \& Terlevich (1995) which, if confirmed, could introduce a systematic effect in the use of the HII galaxies as cosmological probes. Throughout our paper we use $H_{0}=75 \mathrm{~km} \mathrm{~s}^{-1} \mathrm{Mpc}^{-1}$.

\section{Sample selection and methodology}

We consider the original sample of $128 \mathrm{HII}$ galaxies, used to estimate the Hubble constant in Chávez et al. (2012) which was selected from the SDSS DR7 spectroscopic data within a redshift range $0.01<z<0.2$. The sources were chosen for being compact $(D<5$ arcsec) and having large Balmer emission line fluxes and equivalent widths. A lower limit of $50 \AA$ for the $\mathrm{H} \beta$ equivalent width $(W)$ was chosen in order to avoid more evolved starbursts, that would present underlying absorptions due to an older stellar population component, thus affecting the emission lines flux (cf. Melnick et al. 2000).

High resolution echelle spectroscopy was performed at $8 \mathrm{~m}$ class telescopes (Subaru \& VLT) and long slit spectrophotometry at the $2.1 \mathrm{~m}$ telescope of the Observatorio Astronómico Nacional (OAN) in San Pedro Mártir and at the $2.1 \mathrm{~m}$ telescope of the Observatorio Astrofísico Guillermo Haro (OAGH) in Cananea, both in Mexico. Full details of the sample selection, observations and data reduction and analysis are given elsewhere (Chávez et al. 2012; and in prep.).

To identify neighbors around each HII galaxy, within the Sloan Digital Sky Survey (SDSS; DR7), we apply a projected rest-frame maximum radius separation of $<200 h_{75}^{-1} \mathrm{kpc}$, as well as radial velocity limit separation of $\Delta u<600 \mathrm{~km} \mathrm{~s}^{-1}$ (similar to the pairwise galaxy velocity dispersion; e.g. Jing et al. 1998), when spectroscopic redshifts are available, or $\Delta z<0.025$ (i.e., the rms error of the SDSS photometric z's) when only photometry is available. Even though there is no general concensus on the maximum radial separation of a galaxy pair, most of the recent studies use a search radius between $20 h^{-1} \mathrm{kpc}$ (e.g. Patton et al. 2005) and $200 h^{-1} \mathrm{kpc}$ (e.g. Focardi et al. 2006; see also relevant discussion in Deng et al. 2008). We choose the limit of $200 h_{75}^{-1} \mathrm{kpc}$ considering that it is a reasonable distance for a satellite galaxy in a massive halo (e.g. Bahcall et al. 1995; Zaritsky et al. 1997), while this limit is also a compromise between having enough "isolated" and "paired" HII galaxies. Had we increased its value we would reduce greatly the number of isolated galaxies and vice versa.

In addition, we limit our neighbor search to a maximum SDSS $m_{r}$-band magnitude difference between the HII galaxy and its companion of $\Delta m_{r}=+1$, since the SDSS completness limit is $m_{r} \sim 20.5$ while our HII galaxy sample is limited to $m_{r}<19$, reducing our sample to 110 objects.

Our aim is to separate the isolated HII galaxies from those with at least one neighbor within the specified angular and velocity limits, and then compare their physical properties, i.e. their velocity dispersion $\sigma, \mathrm{H} \beta$ emission line luminosity ${ }^{2} L_{\mathrm{H} \beta}$ and metallicity $Z$ (defined as $\mathrm{O} / \mathrm{H}$ abundance).

In our initial analysis we choose to use only the HII galaxies which have neighbors with spectroscopic redshifts (i.e., we exclude all HII galaxies that have neighbors based only on photometric $z$ 's, due to the known relatively large photo- $z$ uncertainty). Note that although the above exclusion of photo- $z$ pairs is expected to reduce the noise in our results, it could introduce a bias towards brighter pairs since the spectroscopic SDSS catalog is complete only to an $r$ magnitude of 17.77 . This bias manifests itself also as a redshift distribution difference between the HII galaxies having a neighbor and the isolated ones. Nevertheless, we will use these results as a starting point. Ideally, we would like to compare subsamples matched in redshift and with available spectroscopy. This is possible at present only for a redshift limited sample up to $z=0.05$, below which there is no redshift distribution difference of the two subsamples. However, in this case the size of the sample is greatly reduced, a fact that may affect the significance of our results.

Therefore, we choose also to use the photo- $z$ based pairs in our analysis, since their inclusion (at the expense of additional noise) eliminates the problem of the uneven redshift distribution between isolated and paired HII galaxies without reducing the respective subsamples. Our goal for the future is to obtain spectroscopic redshifts of all the photo- $z$ based neighbors and confirm our results.

Telles \& Televich (1995) concluded that their sample of HII galaxies with a close companion tends to be more compact and less disturbed than the respective sample of isolated ones. Because of the faint magnitudes and compactness of the majority of our sample galaxies, we are not able to reach a definite conclusion on the latter. We will however investigate the possible role of interactions in the compactness of the HII galaxies. To this end we use the physical diameters of the HII galaxies which are derived from the apparent SDSS isophotal diameters (at $25.0 r$-mag $\operatorname{arcsec}^{-2}$ ). The data and calculation method are available in the NED. We should note here that the Petrosian radius is a better measure of the size of extended sources, but for compact objects the isophotal diameters can also be used. To test the different definitions, we compared the Petrosian to the isophotal diameters of a small random subsample of our HII galaxies and found that they are completely consistent.

\section{Results}

In Fig. 1 we plot the luminosity and velocity dispersion distributions of 62 isolated and 17 paired (using spec-z's and $\Delta m_{r}=$ +1.0) HII galaxies. The KS test indicates that the luminosity and velocity dispersion distributions are significantly different, the former more than the latter (see Table 1), in the sense that

\footnotetext{
2 Throughout when referring to luminosity we will always imply $\mathrm{H} \beta$ luminosity.
} 
E. Koulouridis et al.: The environment of HII galaxies revisited
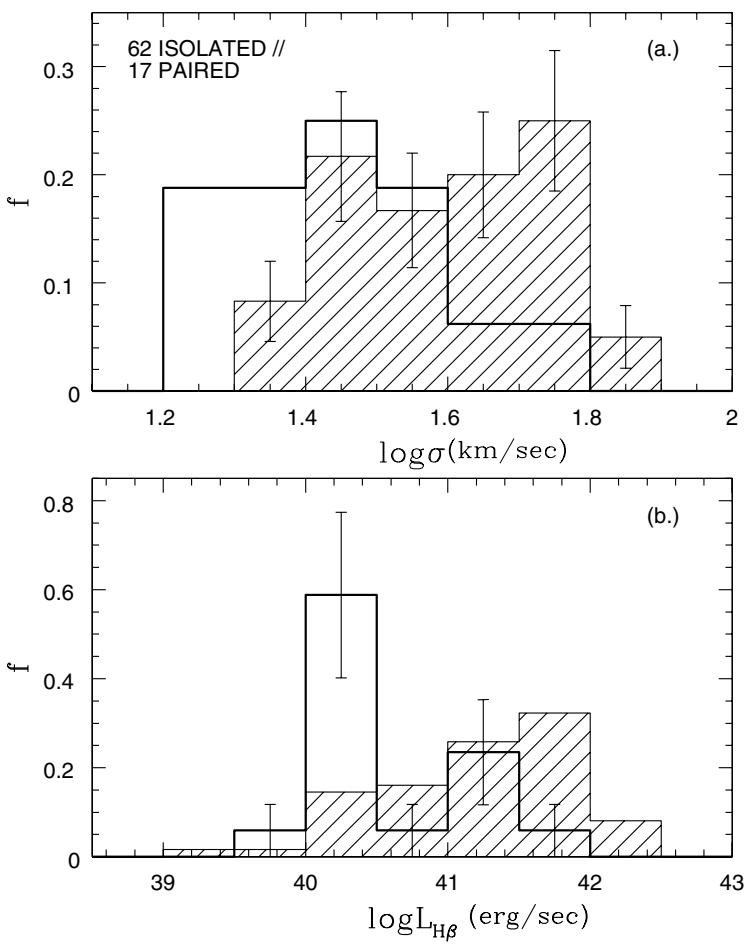

Fig. 1. Velocity dispersion (panel a)) and luminosity (panel b)) distributions of HII galaxies with (plain) and without (hatched) spectroscopically confirmed neighbors. Uncertainties are $1 \sigma$ Poisson errors.

Table 1. Results of Kolmogorov-Smirnov statistical tests.

\begin{tabular}{lccccccc}
\hline \hline$z$ confirmation & $\begin{array}{c}\text { Fig. } \\
(1)\end{array}$ & $\begin{array}{c}z \\
(3)\end{array}$ & $\begin{array}{c}N_{\mathrm{i}} \\
(4)\end{array}$ & $\begin{array}{c}N_{\mathrm{p}} \\
(5)\end{array}$ & $\begin{array}{c}K S_{\sigma} \\
(6)\end{array}$ & $\begin{array}{c}K S_{\mathrm{L}} \\
(7)\end{array}$ & $\begin{array}{c}K S z \\
(8)\end{array}$ \\
\hline Spectroscopy & 1 & 0.15 & 62 & 17 & 0.011 & 0.003 & 0.005 \\
Spectroscopy & 2 & 0.05 & 32 & 14 & 0.098 & 0.030 & 0.129 \\
Spec. and photometry & 3 & 0.15 & 62 & 48 & 0.067 & 0.011 & 0.952 \\
\hline
\end{tabular}

Notes. (1) Redshift confirmation; (2) respective figure number; (3) upper limit of HII galaxy redshift; (4) Number of isolated HII galaxies; (5) Number of HII galaxies with at least one neighbor within $200 h_{75}^{-1} \mathrm{kpc}$; (6) probability that the null hypothesis that the samples are drawn from the same parent population can not be rejected for velocity dispersion distributions; (7) same as Col. 6 for luminosity distributions; (8) same as Col. 6 for redshift distributions.

isolated HII galaxies exhibit higher luminosities and higher velocity dispersions with respect to those having close neighbors. Considering neighbors with $z<0.05$ (Fig. 2) supresses the problem of the uneven redshift distribution between isolated and paired HII galaxies (last column of Table 1) at the cost of reducing greatly the number of isolated HII galaxies. The results remain practically the same, even though less statistically significant (especially for the velocity dispersion). We should note however that this is probably due to the small number of available objects.

By considering in the previous analysis only the spec- $z$ based pairs, to avoid projection effects due to unreliable photometric redshifts, we have excluded more than half our HII galaxy sample resulting in less significant statistical results. Furthermore, by not imposing a redshift limit in Fig. 1, we may have also introduced a bias towards brighter HII galaxy neighbors, as we have discussed earlier.
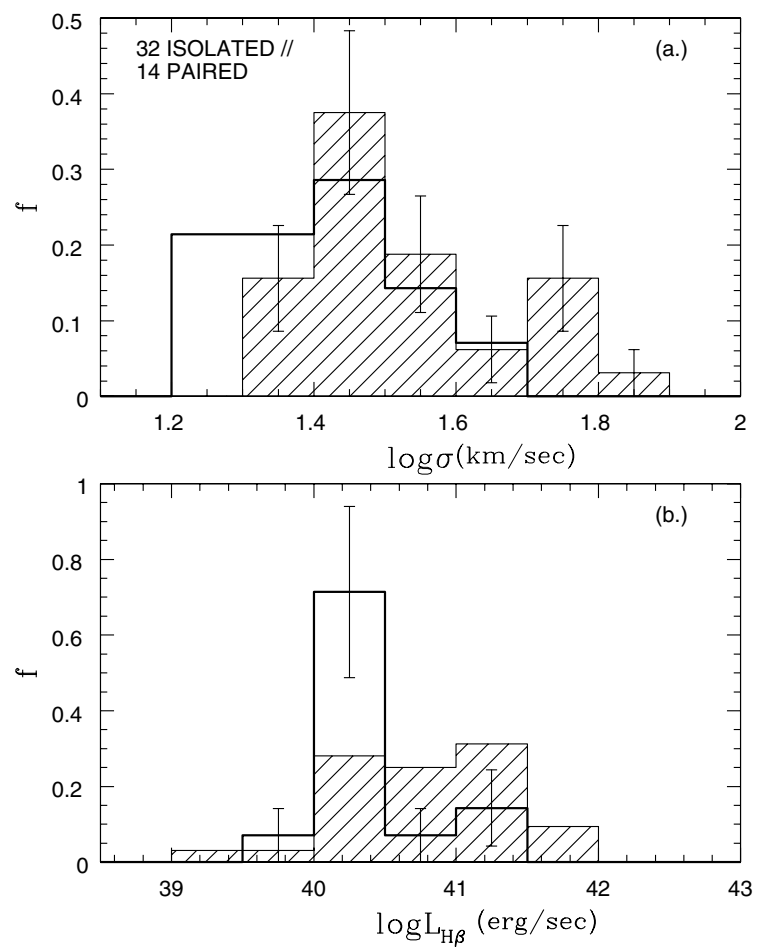

Fig. 2. Velocity dispersion (panel a)) and luminosity (panel b)) distributions of the volume limited HII galaxy sample $(z<0.05)$ with (plain) and without (hatched) spectroscopically confirmed neighbors. Uncertainties are $1 \sigma$ Poisson errors.

We now relax the above conditions and we add neighbors with available photometric redshift within the limit $\Delta m_{r}=+1.0$, considering all photometrically confirmed neighbors as "true" neighbors (Fig. 3). This increases considerably (almost triples) the number of paired HII galaxies (from 17 to 48). Despite the fact that we surely contaminate the subsample of paired HII galaxies with a number of isolated ones because of the greater photometric redshift uncertainty of faint objects, we can see that the luminosity and velocity dispersion distributions can be considered statistically different, but the former at a reduced confidence level with respect to the Fig. 1's case (see Table 1). In addition, we cannot reject the hypothesis that the redshift distributions of the two subsamples are drawn from the same parent population at any significant statistical level.

We should note here that excluding all galaxies with redshift above $z=0.05$ from the analysis, presuming that the photometric redshifts of low $z$ galaxies are more reliable, the trends remain practically the same as for the whole sample.

In all cases the metallicity distributions of the two subsamples are statistically equivalent and therefore we do not present any extra plots.

Returning to the issue of the compactness of the HII galaxies we apply a KS test on the diameter distributions of a low redshift volume limited subsample, where all pairs are spectroscopically confirmed (Fig. 4a), and of the whole parent sample using in addition photometrically confirmed neighbors (Fig. 4b). Once more, we use volume limited subsamples to avoid introducing any distance dependent bias. We conclude that although we cannot reject the null hypothesis that the two aforementioned distributions are drawn from the same parent population at any significant confidence level, we observe a difference between them which mainly arises from a visible shift. This shift is due to the 

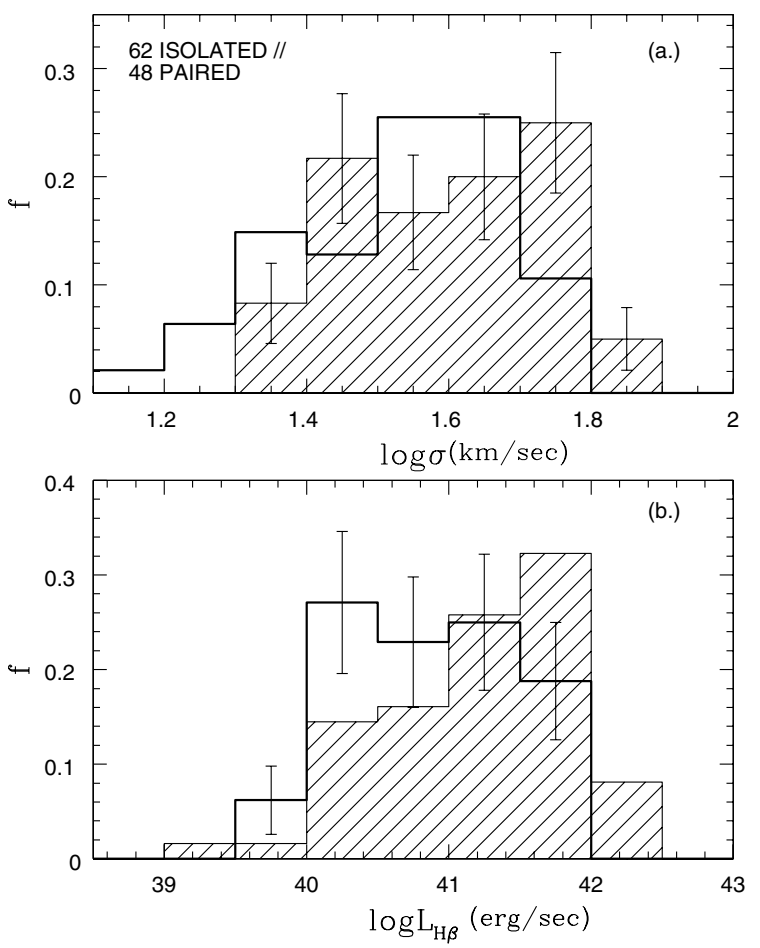

Fig. 3. Velocity dispersion (panel a)) and luminosity (panel b)) distributions of HII galaxies with (plain) and without (hatched) spectroscopically or photometrically confirmed neighbors. Uncertainties are $1 \sigma$ Poisson errors.

more compact objects being mostly HII galaxies with neighbors, while the more extended ones are isolated. We should note here that by investigating the diameter distributions of the HII galaxies in a small number of narrow redshift bins, we find that this effect persists in each bin. This partially confirms the results of Telles \& Terlevich (1995) who found a weak trend where the most compact HII galaxies tend to have a close neighbor, whereas the most extended ones tend to be isolated. However, this trend between the two subsamples is not highly significant and a larger sample would be necessary in order to confirm it.

We now wish to investigate whether the particular systematic effect that we have identified, i.e., the environmental dependence of both the $\mathrm{H} \beta$ luminosity and the velocity dispersion, affects the cosmologically relevant $L_{\mathrm{H} \beta}-\sigma$ correlation. Qualitatively one should not expect any important effect on the correlation since the existence of a close neighbor affects both the $\mathrm{H} \beta$ luminosity and $\sigma$ in the same direction; i.e., they both decrease following the monotonic trend of the observed correlation. Nevertheless, to be more quantitative, we fit the above correlation separately for the isolated or the paired HII galaxies, and we compare the slopes of the correlation with that of the whole sample together. In Fig. 5 we plot the three lines which best fit each sample (black line for the parent sample (all points), red line for the paired HII sample (open squares) and green line for the isolated sample (triangles)). The dashed lines define the confidence band at $2 \sigma$ confidence level of the parent sample's regression line, taking the joint distribution of the slope and the intercept into account.

For the case of isolated HII galaxies we find a relative difference of the slopes of the $L_{\mathrm{H} \beta}-\sigma$ relation $\delta a / a_{\mathrm{T}}=\left(a_{\mathrm{isol}}-\right.$ $\left.a_{\mathrm{T}}\right) / a_{\mathrm{T}}=-0.06 \pm 0.10$, while for the case of paired HII galaxies, $\delta a / a_{\mathrm{T}}=0.08 \pm 0.13$, where $a_{\mathrm{T}}$ is the slope of the correlation based on the parent sample of HII galaxies.
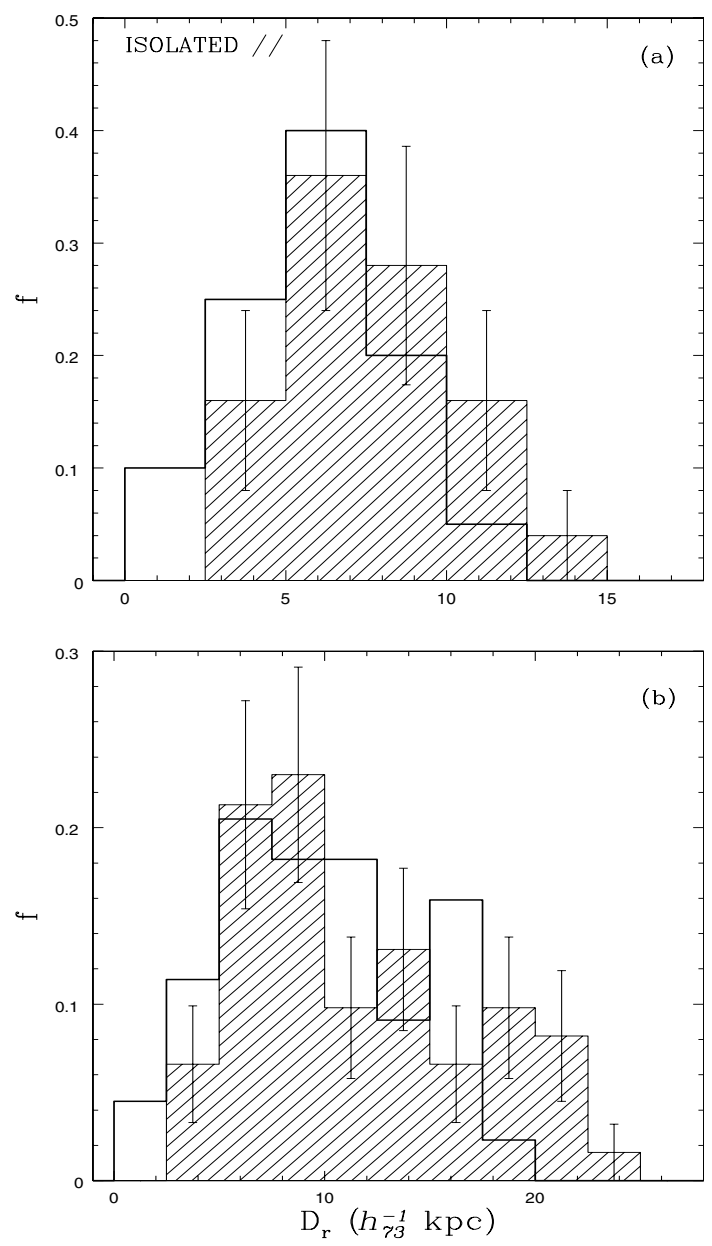

Fig. 4. Isophotal r-SDSS diameter distribution of isolated (hatched area) and paired HII galaxies of a) a low redshift $(z<0.05)$ volume limited subsample of HII galaxies with spectroscopically confirmed neighbors, and b) of the whole parent sample using also photometrically confirmed neighbors. Uncertainties are $1 \sigma$ Poisson errors.

In an attempt to determine if any discrepancies can also arise due to random sampling of 62 or 48 objects drawn from the parent population independently of their properties, the errors were calculated by applying the bootstrap method i.e. by resampling randomly each subsample from the parent sample. Indeed, in both cases, we find no significant difference.

This is indeed a very important result, indicating that although environmental effects do influence the dynamics of the starburst they do not affect the cosmologically important $L_{\mathrm{H} \beta}-\sigma$ correlation. Given the size of the sample, the possibility remains that these small differences between the slopes of the two subsamples are intrinsic. However, to verify if such small slope differences are real, we would need at least to triple the number of objects, a difficult task considering the expensive observational requirements for this kind of studies. Furthermore, such a difference in the slope of the relation is not found even at much higher densities, as shown by the similarity of the slope of the L-sigma relation for Giant HII regions in the disks of massive spirals (see Sect. 4).

\section{Conclusions and discussion}

We have studied a sample of 110 HII galaxies which was selected from the SDSS DR7 spectroscopic data within a redshift 


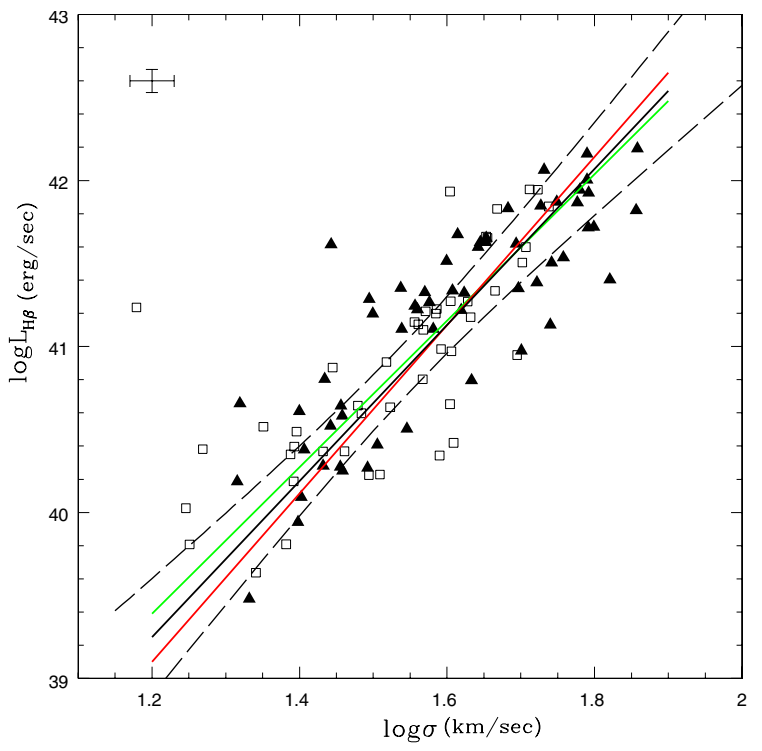

Fig. 5. $L-\sigma_{\mathrm{H} \beta}$ diagram. The red line denotes the linear regression fitting of the isolated HII galaxies (triangles), while the green line denotes the fitting of those that have at least one spectroscopically or photometrically confirmed neighbor within $200 h_{75}^{-1} \mathrm{kpc}$ (squares). The black line is the fitting of the parent sample and the dashed lines define the $2 \sigma$ confidence band of the regression line. Typical $1 \sigma$ errors are shown on the upper left corner of the plot.

range $0.01<z<0.16$ and $m_{r}<19$. Our results indicate that there is a connection between the existence of a companion and the size of the star forming region.

In particular we find that both The $\mathrm{H} \beta$ luminosities and velocity dispersions of the HII galaxies with neighbors (within a projected rest-frame radius separation of $<200 h_{75}^{-1} \mathrm{kpc}$ and radial velocity separation of $\Delta u<600 \mathrm{~km} \mathrm{~s}^{-1}$ ) tend to be significantly lower than those of the isolated ones.

Importantly, the $L_{\mathrm{H} \beta}-\sigma$ correlation and distance estimator is not affected by the environmental or host galaxy differences of the isolated and paired HII galaxies. One would like to understand the physical mechanism by which the environmental dependence of $L_{\mathrm{H} \beta}$ and $\sigma$ is such as to leave unaffected the correlation. If this correlation is a manifestation of the virial theorem, it is evident that the mass of the molecular cloud, progenitor of the massive starburst, is influenced by the environment, i.e. more massive clouds, producing more massive starbursts and therefore higher values of $L_{\mathrm{H} \beta}$ and $\sigma$, are developed in lower density environments, while the lower mass clouds are characteristic of higher density environments, where more frequent interactions can limit the growth of the molecular clouds. This is even more evident when including in the discussion the results of Giant HII regions in the disks of massive spirals. Giant HII regions are massive bursts of star formation in a much higher density environment than that of HII galaxies. As can be seen from the analysis of Melnick et al. (1988) or Chávez et al. (2012), Giant HII regions and HII galaxies define a tight $L_{\mathrm{H} \beta}-\sigma$ correlation where Giant HII regions occupy the lower end of the relation; i.e. they tend to be much smaller than HII galaxies while independently verifying the same tight relation between $L_{\mathrm{H} \beta}$ and $\sigma$ of the more luminous HII galaxies notwithstanding the fact that they are formed in a much denser environment.

Our results agree with previous studies showing that HII galaxies are less clustered than normal galaxies (e.g. Iovino et al. 1988; Loveday et al. 1999; Telles \& Maddox 2000), and that they lack massive neighbors (Campos-Aguilar \& Moles 1991; Campos-Aguilar et al. 1993). Even considering all photometrically confirmed neighbors (faint neighbors) as real neighbors, more than half of our sample galaxies remain isolated. Our results therefore concur with the view where star formation in HII galaxies is not necessarilly triggered by interactions (Telles \& Terlevich 1995; Telles \& Maddox 2000); they do appear however to play an important role in the confinement of the total mass of the progenitor molecular cloud that gives birth to an HII galaxy. Although the triggering mechanism of the enhanced star forming activity of HII galaxies is still debated, star formation is probably bound to happen when even an isolated molecular cloud fulfills the requirements (see discussion in Telles 2010). Alternatively, and since older stellar populations have been found to be present (e.g. Papaderos et al. 1996; Telles \& Terlevich 1997; Cairós et al. 2003), implying that not all HII galaxies are young formations, it could also be a manifestation of cosmic downsizing; less massive structures are unable to efficiently form stars in the past and they are doing so in later epochs, when most massive structures are already quiescent (e.g. Neistein et al. 2006). Thus, given that star formation in most HII galaxies happens spontaneously and depends only on the mass of the already virialized system, the $L_{\mathrm{H} \beta}-\sigma$ relation should be expected as well.

Not all HII galaxies should be expected to verify the $L_{\mathrm{H} \beta}-\sigma$ relation with a small scatter. Already Melnick et al. (1988) had recognized that systems with $\log \sigma>1.75$ show a flattening of the relation, probably indicating the onset of rotation for larger star forming regions, and that limiting the sample to objects with $10 \mathrm{~km} \mathrm{~s}^{-1}<\sigma<60 \mathrm{~km} \mathrm{~s}^{-1}$, equivalent widths $W_{\mathrm{H} \beta}>50 \AA$, and Gaussian profiles in their emission lines, produces a tight $L_{\mathrm{H} \beta}-\sigma$ relation, suggesting that we are dealing with young massive bursts that dominate the luminosity of the galaxy and that they are gravitationally bound and pressure supported. The biases introduced by multiplicity, rotation, and contamination by the underlying galaxy (e.g. Overzier et al. 2008; Amorin et al. 2012) are minimized by selecting only objects with emission lines of high equivalent width and line profiles that are well fitted by a single Gaussian.

\section{References}

Amorín, R., Vílchez, J. M., Hägele, G. F., et al. 2012, ApJ, 754, L22 Bahcall, N. A., Lubin, L. M., \& Dorman, V. 1995, ApJ, 447, L81 Bekki, K. 2008, MNRAS, 388, L10

Bordalo, V., \& Telles, E. 2011, ApJ, 735, 52

Bosch, G., Terlevich, E., \& Terlevich, R. 2002, MNRAS, 329, 481

Brosch, N., Bar-Or, C., \& Malka, D. 2006, MNRAS, 368, 864

Cairós, L. M., Caon, N., Papaderos, P., et al. 2003, ApJ, 593, 312

Campos-Aguilar, A., \& Moles, M. 1991, A\&A, 241, 358

Campos-Aguilar, A., Prieto, M., \& Garcia, C. 1993, A\&A, 276, 16

Chávez, R., Terlevich, E., Terlevich, R., et al. 2012, MNRAS, 425, L56

Deng, X.-F., He, J.-Z., Jiang, P., Song, J., \& Tang, X.-X. 2008, ApJ, 677, 1040

Ellison, S. L., Patton, D. R., Simard, L., \& McConnachie, A. W. 2008, AJ, 135, 1877

Focardi, P., Zitelli, V., Marinoni, S., \& Kelm, B. 2006, A\&A, 456, 467

Fuentes-Masip, O., Muñoz-Tuñón, C., Castañeda, H. O., \& Tenorio-Tagle, G. 2000, AJ, 120, 752

Ideue, Y., Taniguchi, Y., Nagao, T., et al. 2012, ApJ, 747, 42

Iovino, A., Melnick, J., \& Shaver, P. 1988, ApJ, 330, L17

Jing, Y. P., Mo, H. J., \& Boerner, G. 1998, ApJ, 494, 1

Koulouridis, E., Chavushyan, V., Plionis, M., Krongold, Y., \& Dultzin-Hacyan, D. 2006, ApJ, 651, 93

Li, C., Kauffmann, G., Heckman, T. M., White, S. D. M., \& Jing, Y. P. 2008 , MNRAS, 385, 1915

Melnick, J., Terlevich, R., \& Moles, M. 1988, MNRAS, 235, 297

Melnick, J., Terlevich, R., \& Terlevich, E. 2000, MNRAS, 311, 629

Neistein, E., van den Bosch, F. C., \& Dekel, A. 2006, MNRAS, 372, 933 
A\&A 554, A13 (2013)

Noeske, K. G., Iglesias-Páramo, J., Vílchez, J. M., Papaderos, P., \& Fricke, K. J. 2001, A\&A, 371, 806

Papaderos, P., Loose, H.-H., Thuan, T. X., \& Fricke, K. J. 1996, A\&AS, 120 207

Patton, D. R., Grant, J. K., Simard, L., et al. 2005, AJ, 130, 2043

Overzier, R. A., Heckman, T. M., Kauffmann, G., et al. 2008, ApJ, 677, 37

Plionis, M., Terlevich, R., Basilakos, S., et al. 2011, MNRAS, 416, 2981

Riess, A. G., Macri, L., Casertano, S., et al. 2011, ApJ, 730, 119

Sanders, D. B., \& Mirabel, I. F. 1996, ARA\&A, 34, 749

Siegel, E. R., Guzmán, R., Gallego, J. P., Orduña López, M., \& Rodríguez Hidalgo, P. 2005, MNRAS, 356, 1117
Surace, J. A., Sanders, D. B., Vacca, W. D., Veilleux, S., \& Mazzarella, J. M. 1998, ApJ, 492, 116

Telles, E. 2010, Galaxy Wars: Stellar Populations and Star Formation in Interacting Galaxies, 423, 65

Telles, E., \& Terlevich, R. 1995, MNRAS, 275, 1

Telles, E., \& Terlevich, R. 1997, MNRAS, 286, 183

Telles, E., Melnick, J., \& Terlevich, R. 1997, MNRAS, 288, 78

Telles, E., Muñoz-Tuñón, C., \& Tenorio-Tagle, G. 2001, ApJ, 548, 671

Terlevich, R., \& Melnick, J. 1981, MNRAS, 195, 839

Vilchez, J. M. 1995, AJ, 110, 1090

Zaritsky, D., Smith, R., Frenk, C., \& White, S. D. M. 1997, ApJ, 478, 39 